\title{
Functional liposomes and supported lipid bilayers: towards the complexity of biological archetypes
}

\author{
Debora Berti, ${ }^{a b}$ Gabriella Caminati $^{a b}$ and Piero Baglioni ${ }^{* a b}$ \\ Received 4th November 2010, Accepted 28th February 2011 \\ DOI: $10.1039 / \mathrm{c0cp} 02400 \mathrm{~g}$
}

This perspective paper provides some illustrative examples on the interplay between information gathered on planar supported lipid bilayers (SLB) and unilamellar lipid vesicles (ULV) to get an integrated description of phenomena occurring at the nanoscale that involve locally bilayered structures. Similarities and differences are underlined and critically compared in terms of biomimetic fidelity and instrumental accessibility to structural and dynamical parameters, focusing on some recent reports that either explicitly address this comparison or introducing some studies that separately investigate the same process in SLB and lipid vesicles. Despite the structural similarity on the nanoscale, the different topology implies radically different characterization techniques that have evolved in sectorial and separated approaches. The quest for increasing levels of compositional complexity for bilayered systems should not result in a loss of structural and dynamical control: this is the central challenge of future research in this area, where the integrated approach highlighted in this contribution would enable improved levels of understanding.

\section{Introduction}

Despite the fact that the first investigations on self-assembled lipid systems date back almost to half a century ago, ${ }^{1}$ the field of lipid

${ }^{a}$ Department of Chemistry and CSGI, University of Florence, Via della Lastruccia 3-Sesto Fiorentino, 50019 Florence, Italy. E-mail:baglioni@csgi.unifi.it; Fax:0039 0554573032;

Tel: 00390554573033

${ }^{b}$ CSGI, Via della Lastruccia 3, 50019 Sesto Fiorentino, Firenze, Italy. Fax: 0039 0554573036; Tel: 00390554573034 bilayers is still an active research area. ${ }^{2}$ The fertility of the topic is motivated by the many and diverse applications envisaged for a variety of purposes, from drug delivery ${ }^{3}$ to gene therapy, ${ }^{4}$ to sensors, ${ }^{5}$ to confined-environment reactors. ${ }^{6}$ The structural similarity of vesicles with cell membranes has motivated the development of lipid bilayers as host model systems for intrinsic membrane proteins and as simpler mimics for fundamental studies of membrane-mediated biomolecular interactions. ${ }^{7}$

As the available techniques and the knowledge on lipid structure and function have enormously progressed throughout

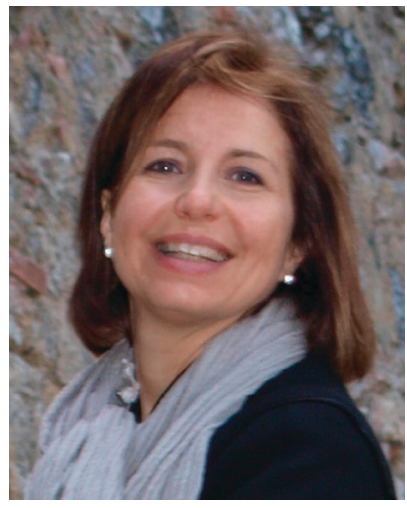

Debora Berti
Debora Berti studied Chemistry at the University of Florence, where she obtained her doctoral degree in Chemical Sciences in 1997. She has been Visiting Scientist at the ETH (Zurich) during 1994-1995 and postdoctoral fellow for CSGI (Italian Center for Colloid Science) until 2000. In 2000 she joined the University of Florence, where she works on phospholipid and nucleolipid self-assembly and teaches Physical Chemistry of Nanosystems to undergraduate and graduate Chemistry and Biotechnology students. She is the author of more than 65 research papers and has presented more than 50 invited and contributed lectures to international conferences and meetings.

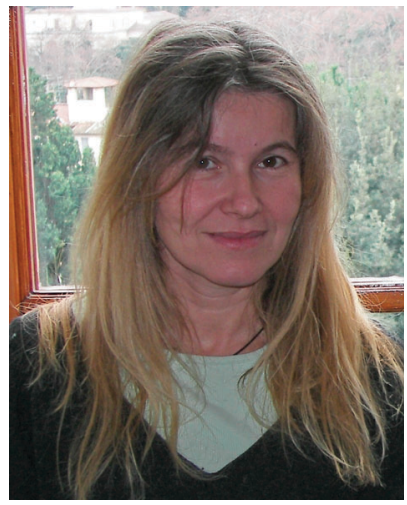

Gabriella Caminati received her doctoral degree in Chemical Sciences in 1990 from the University of Florence. She was a postdoctoral researcher at Colombia University of New York and a Visiting Scientist at the Max-Planck Institute für Biophysikalische Chemie in Göttingen and at the Ecole Normale Supérieure de Lyon. In 1991 she joined the University of Florence where she teaches Physical Chemistry of Molecularly Organized Systems. Her research interests span from physical chemistry of micro-heterogeneous and interfacial systems to photophysics in confined environments with special focus on nanorganized stuctures for sensing and electroptical devices. 
these decades, novel opportunities have emerged to design, characterize and apply lipid-bilayered structures.

Two alternative approaches are commonly employed to determine structural and dynamical information: in the first one supported samples (either single or multibilayers) are used, while in the second approach multilamellar or unilamellar freestanding bilayers are investigated. In the case of supported single or multibilayers the interaction between the substrate and the lipid bilayers can alter their physical state, with maximum perturbation for the bilayer adjacent to the support. For both kinds of multibilayer samples (supported or free standing), possible effects of interbilayer interactions must be taken into account. On the other side, classical small angle diffraction experiments, methods of choice to investigate bilayer systems, benefit from the increased amount of sample material present in multilamellar systems. ${ }^{8}$ However in some situations, the multibilayer approach is not applicable. In particular, when interactions between bilayers and amphiphilic or water-soluble molecules are to be investigated, or when these binding steps initiate other cascade or sequential events, it is preferable, if quantitative information is needed, to rely on single-bilayer models. The multibilayer approach can also result from difficult applications when the interaction involves changes in the surface charge density, leading to an increase in the repulsive energy between adjacent bilayer that can be difficult to decouple from the information of interest.

Neither ULV (unilamellar lipid vesicles) nor SLB (supported lipid bilayers) can be considered per se the optimal, standalone model system, as we will briefly outline in the next paragraph. In the first case, the effects of curvature, which is much higher with respect to cell bilayers, might play a role, while in the second case the substrate might affect the structural and dynamical fidelity with respect to free standing bilayers.

In this Perspective, we will bring evidence of how the comparison between functional ULV and SLB systems, with identical nanoscale order but different mesoscopic order, can provide improved understanding and complementary information for applications on bio-relevant research areas.

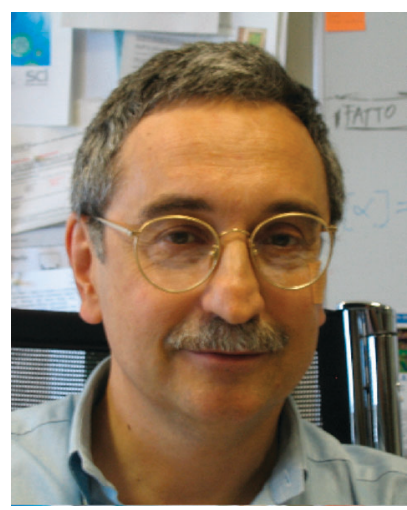

Piero Baglioni
Piero Baglioni is currently Full Professor of Physical Chemistry in the Department of Chemistry of the University of Florence. He is also the Director of the Italian National Consortium for Nanosystems (CSGI). He is the author of more than 300 publications and 18 patents in the field of colloids and interfaces and a pioneer in the application of soft matter to new high concentration suspensions, phototherapy and diagnosis of tumors, conservation of cultural heritage, production of emulsions from Bio Crude Oil, production of nanoparticles and novel nano-coatings via flame-spraying.
The contribution is organized as follows: first the main structural features of the bilayer scaffolds are introduced, referring the readers to more detailed reviews on preparative protocols and applications; the main characterization techniques are briefly mentioned and referenced, with particular emphasis on possible complementary experimental approaches. Then, some case studies will be presented, where bilayers with different topologies are introduced to study similar problems or either conceived to explicitly address the comparison between liposomes and SLB.

\section{3D Scaffolds: lipid unilamellar vesicles}

Phospholipid vesicles, or liposomes, are bilayered closed shells dispersed in aqueous medium enclosing a pool of solvent in their interior. They can be prepared by dispersing the thermodynamically stable lipid lamellar phase, ${ }^{9}$ where flat infinite bilayers, composed of oppositely oriented monolayers, are separated by a water layer, with a smectic period dictated by the maximum swelling. ${ }^{10}$

The basic structural motif is a bilayer where two molecular building blocks, i.e. lipid molecules, arrange themselves in a tail-to-tail fashion, exposing their polar head to the aqueous environment. The driving force for spontaneous self-assembly into bilayers stems from the amphiphilic nature of lipids and is therefore due to hydrophobic effects. ${ }^{11}$ Bilayers have generally zero spontaneous curvature, ${ }^{12}$ and their bending requires a considerable Gibbs energy contribution. Different experimental protocols for the preparation of liposomal dispersions from lamellar phases have been proposed throughout the years, and generally involve the input of mechanical energy, like treatments with ultrasonic baths or vortex-mixing followed by membrane extrusion..$^{13} \mathrm{~A}$ review on preparation methods is beyond the aims of this contribution, and the reader is referred to the extensive literature already present on the subject. ${ }^{14}$ During these processes, the swollen bilayers are fragmented into small bilayer patches, whose edges with hydrophobic chains are exposed to water. Beyond a critical radius, the disks will close and form vesicles. Vesicle formation, in this case, results from a balance between the disk edge energy and the bending energy of the bilayer, which will favour this second event. Liposomes are kinetic locks, with intrinsic metastability, and will eventually aggregate and return to the equilibrium lamellar phase in excess solvent.

Fig. 1 sketches the chemical structure of some typical lipids, among the predominant species in natural membranes, together with the assembly pattern in lamellar phases and liposomes.

Currently, several methods are available to prepare liposomal systems with controlled composition, size, charge, membrane fluidity and radius polydispersity, which can be reasonably narrow, depending on the desired size.

Liposomes are often categorized according to lamellarity (i.e. unilamellar vs. multilamellar) or size (SUVs, small unilamellar vesicles, with diameters ranging from 30 to $100 \mathrm{~nm}$; LUVs, large unilamellar vesicles, from 100 to $500 \mathrm{~nm}$, GUVs giant unilamellar vesicles, with diameters of several microns). Despite this large spread in size, the bilayer thickness, i.e. the shell size, is directly connected to molecular size and 


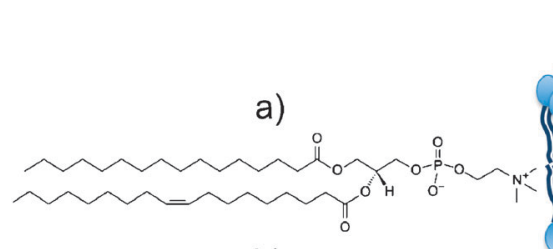

b)

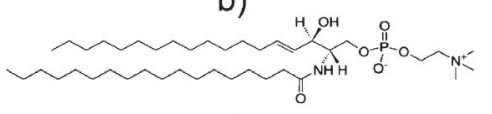

c)

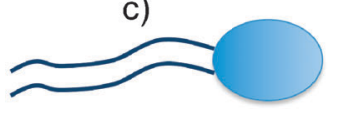

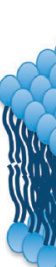

(1). (1) (j)

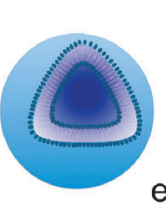

d)

e)
Fig. 1 Chemical structure of two representative phospholipids: 1-palmitoyl-2-oleoyl-sn-glycero-3-phosphocholine (a); and $\mathrm{N}$-stearoyl-D-erythro-sphingosylphosphorylcholine (b). They can be represented as amphiphilic molecules (c), whose self-assembly results in planar bilayers (d) and liposomes (e).

is generally around $4 \mathrm{~nm}$. It has been observed ${ }^{14}$ that the minimum radius of liposomes is roughly 3-5 times the bilayer thickness; below this threshold the spontaneous curvature of the bilayer would be frustrated by the liposomal curvature and bilayers would not bend.

For large or giant vesicles, it is generally accepted that the vesicle diameter has little effect on the local structure of the bilayer, since the characteristic sizes, i.e. the molecular size and the radius of curvature of the liposomes are at least two orders of magnitude apart: this means that locally each lipid molecule experiences a flat curvature, as in the planar lamellar phase.

The lipid bilayer encloses an aqueous compartment, which can have identical or different composition as the external milieu, i.e. it can withstand concentration gradients between its two sides; the permeability to water requires however osmotic balance between the solvent pool and the dispersing medium, to guarantee structural stability against swelling or shrinking.

Since their first observation by Bangham, liposomes have found widespread application as synthetic carriers for drug delivery, for instance encapsulating hydrophilic molecules inside their aqueous core and protecting them from enzymatic inactivation. Size-exclusion chromatography is then commonly employed to separate liposomes loaded with water-soluble cargos in the internal solvent pool, from the same molecules dispersed in the external solution. ${ }^{15}$ Moreover liposomes can confine lipophilic insoluble or amphiphilic drugs in the hydrophobic core, constituted by the lipid apolar chains.

Natural and synthetic lipids are generally zwitterionic or acidic: the lipid composition will then modulate surface charge. Therefore, besides the already-mentioned binding options, several ions or globally charged macromolecules can peripherally bind liposomal surfaces thanks to electrostatic interactions. $^{16}$

In some cases the binding driving force is a combination of the above contributions, as for instance in the case of charged species with some amphiphilic character, like some common proteins.

\section{2D-Scaffolds: supported lipid bilayers}

Supported lipid bilayers (SLB) are among the most popular models of cell membranes with potential biotechnological applications since their introduction in the early 80 s by McConnell. ${ }^{17}$ Phospholipids in SLB are arranged on a planar solid support in a tail-to-tail fashion similar to the liposome bilayer, but while the outer layer is facing directly the surrounding water phase, the inner layer is generally separated from the solid surface by only a thin layer of water (1-2 nm) as depicted in Fig. 2.

Supported phospholipid bilayers can be obtained with a variety of techniques, each of which determines a bilayer system with features that can be very different from each other. Recent reviews ${ }^{18-20}$ nicely describe these methods that can be grouped in 3 principal categories:

(i) Langmuir-Blodgett (LB) methods, either alone or combined with Langmuir-Schafer horizontal deposition.

(ii) Vesicle fusion on a naked solid substrate.

(iii) Vesicle fusion on a pre-covered solid substrate.

In the latter category, the literature describes examples of vesicles ruptured on $\mathrm{LB}^{21}$ or SAMs (self assembled monolayer) layer $^{22}$ and, more interestingly for biological relevant issues, on polymer cushion or hydrogels. ${ }^{23}$ In fact, as reported ${ }^{24}$ in an extensive review on polymer-supported SLB, a loose network of hydrated polymers may serve as a spacer between lipids and substrate avoiding undesired interactions with the substrate causing phase transition or delamination.

In parallel, a multitude of more ambitious architectures have been proposed during the past decade spanning from hybrid bilayers involving vesicle fusion combined with LB or SAM approaches ${ }^{25}$ to tethered lipid bilayers, ${ }^{26,27}$ suspended lipid bilayers or supported vesicular layers. Nevertheless, vesicle fusion on bare substrates is certainly the most extensively studied procedure, although the quest for a mimetic model of intracellular crowding certainly invites the adoption of a cushioned SLB.

A schematic description of the process leading to SLB formation by vesicle spreading is reported in Fig. 3 .

The common pathway (Fig. 3a) of SLB-formation includes vesicle adsorption at the surface followed by vesicles flattening and/or crowding at the surface. Surface adsorbed vesicles eventually rupture and spread on the solid support under the influence of the cooperative action of neighbouring vesicles.

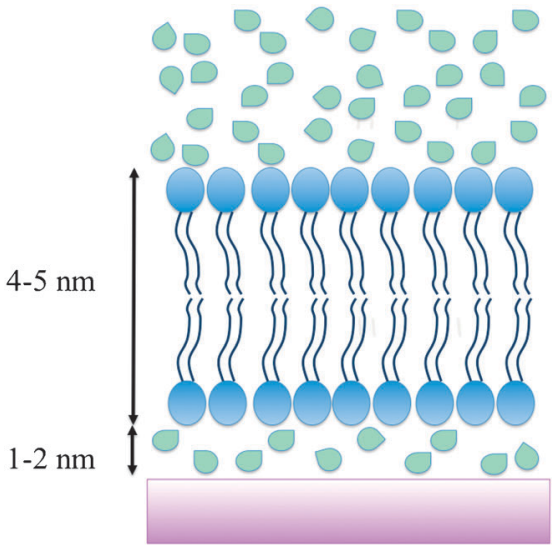

Fig. 2 Schematic diagram of a supported phospholipid bilayer. 


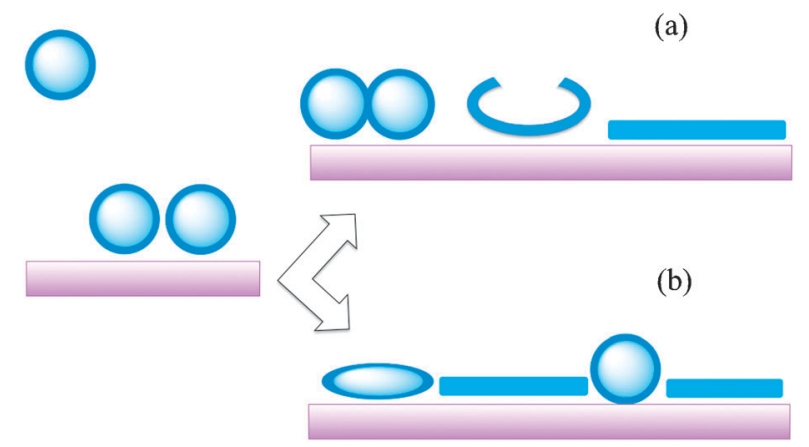

Fig. 3 Schematic description of possible processes leading to SLB formation by vesicle spreading. (a) Vesicle rupture followed by formation of a supported lipid bilayer. (b) Formation of disks and entrapped vesicles.

Vesicle break-up is further propagated by "active edges" of existing bilayer patches and developing bilayer formation.

Nevertheless, the steps leading to the final equilibrium structure may be more complex than depicted in the picture in Fig. 3 and the mechanism of vesicle fusion continues to be studied extensively by many groups. ${ }^{25,28}$ Thanks to these research efforts, the qualitative understanding of SLB formation after exposure of small lipid vesicles to a hydrophilic support is now revealing a stunning variety of effects that can take place during the self-organization process including layer interdigitation, bilayer nano-disk formation, layer-buckling and vesicle trapping in the bilayer (see Fig. 3).

The ensemble of results from Brisson and coworkers has revealed unprecedented insight into intermediates of the SLB-formation process and has helped to identify a number of parameters that are determinant for the final architecture of the lipid bilayer deposition on solid supports including, electrolyte and phospholipid concentration, besides the vesicle lipid composition and surface properties of the solid support.

SLB obtained through vesicle fusion offer many advantages to an experimentalist, including ease of preparation, but one of the most attractive feature of the SLB layout is its remarkable stability. SLB will remain largely intact even when subject to high flow rates or vibration and, unlike black lipid membranes (BLM), the presence of holes will not destroy the entire bilayer. Because of this stability, long lasting experiments are feasible with supported bilayers as opposed to free-standing bilayers BLM, whose existence is usually limited to hours.

Moreover, in SLB systems only the upper face of the bilayer is exposed to the solution; for these systems adsorption and penetration phenomena of molecules dissolved in the water phase can rely on the precise control of the phospholipid molecular density and bilayer thickness. At the same time the fluidity of the bilayer can be tuned by the judicious choice of the phospholipid and the ionic content of the liposome solution regulating the access and penetration of the hydrophobic molecules inside the bilayer.

\section{Structural and dynamical properties and techniques of investigation}

This paragraph focuses on the introduction and comparison between the experimental techniques usually employed to characterize SLB and liposomes from a structural and dynamical point of view. The completeness of picture that can emerge from studying processes in the two bilayer systems is mainly related to the characteristics of the techniques, i.e. surface and classical solution techniques. A careful consideration of the experimental sensitivity and of the assumptions beneath data interpretation is therefore of utmost importance.

The structural characterization of lamellar lipid phases and liposomes can be considered a mature field. As for many other colloidal systems, with relevant lengths at the nanoscale, structural characterization can be performed through imaging techniques in real space, mainly EM and AFM, or alternatively through scattering experiments, where information is obtained in the reciprocal space of scattering vectors. ${ }^{8}$ For dispersed bilayer shells, once the composition and the concentration is set, the structural parameters are the liposome radius, the polydispersity and the bilayer thickness and internal structure. Scattering methods are non-invasive and can be directly applied to liposomal solutions.

Dynamic Light Scattering ${ }^{29}$ is a rather widespread technique, commonly employed to infer the hydrodynamic radius and the polydispersity. For relatively monodisperse and monomodal distribution, the decay in correlation of the scattered light intensity at a given angle can be modelled through a cumulant expansion. ${ }^{30}$ Through this analysis the hydrodynamic radius, which is the actual geometrical radius, can be obtained. The second moment of the cumulant distribution is connected to the polydispersity index that can be used to evaluate the distribution of guest molecules among the liposomal host. For bimodal distributions, each population is weighted by its intensity contribution (i.e. weighted by a $\mathrm{R}^{6}$ factor), and therefore the size distributions obtained by Laplace inversion methods, are generally skewed towards larger size, than the actual number distribution. ${ }^{31}$

The knowledge of the hydrodynamic radius of liposomes is not only important for the above considerations on guest distribution, but also for the fact that its variations when amphiphilic guests are inserted, can be connected to an added hydrodynamic thickness, directly comparable with results on planar bilayers. ${ }^{32}$ This is a very sensitive approach to detect the thickness of the hydrophilic corona, and its changes due for instance to re-orientation of guest molecules as function of grafting density, as illustrated schematically in Fig. 4 and detailed in the first case study of section 6 .

Structural information can be retrieved from Small Angle X-Ray or Neutron Scattering methods (SAXS and SANS), which are well-established techniques for the study of lipid assemblies structures in a range of length scales from around one to one hundred nanometres. The window of scattering vectors probed by these latter techniques allows gathering higher resolution information with respect to light scattering, on the local structure of vesicles, i.e. on the bilayer profile. ${ }^{8}$ The scattering data in the reciprocal space are connected by Fourier transform to the structure in real space (i.e. the electronic density profile in the case of X-Ray, or neutron scattering length density profile, related to chemical composition, for neutrons).

The differences in scattering length densities between the hydrocarbon core, the hydrophilic shell and the solvent, 

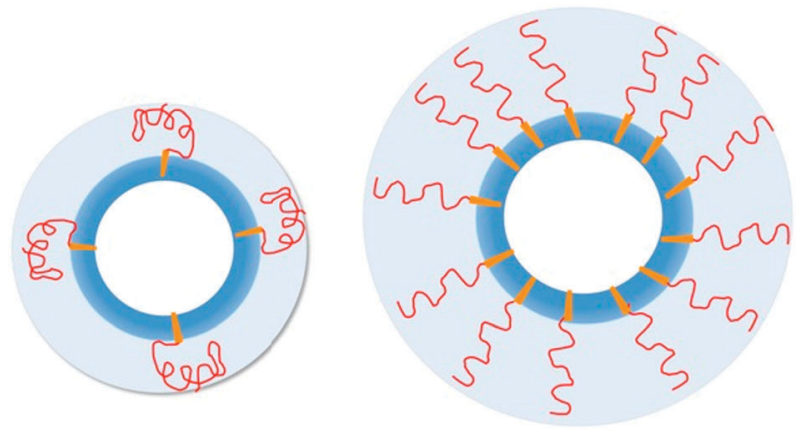

Fig. 4 Variation in hydrodynamic thickness as the grafting density is increased, connected to conformational variation of the guest in the hydrophilic corona.

allow in principle, determination of the structural parameters separately for each of these subdomains of the self-assemblies, provided the spatial resolution (i.e. $\pi / q_{\max }$, with $q$ the highest scattering vector in the experimental range) is sufficient. Moreover several data analysis methods enable the consideration of compositional asymmetry of the two leaflets. The contrast is not particularly favourable for X-rays, while in SANS, thanks to isotopic substitution of $\mathrm{H}_{2} \mathrm{O}$ with $\mathrm{D}_{2} \mathrm{O},{ }^{33}$ hydrocarbon hydrogenated chains provide enhanced contrast. We should here point out, that, even if a SAXS apparatus is more easily accessible as a in-house facility, SANS, with the possibility of isotopic substitution, is particularly indicated especially in the case of lateral domains of different composition, when isotopically labelled components are added.

As stated above, the peculiar planar architecture of supported bilayers and their stability make these systems amenable to a number of characterization tools, which would be impractical if performed on a freely floating sample or on spherical lipid vesicles. Several modern techniques have been used for the characterization of SLB including spectroscopic techniques such as Fourier-transform infrared spectroscopy, ${ }^{34}$ X-ray and neutron scattering approaches, ${ }^{35}$ scanning probe methods ${ }^{36}$ or surface specific techniques as TOF-SIMS, ${ }^{37}$ ellipsometry and surface plasmon resonance, ${ }^{38}$ to cite a few. All these techniques were proven to be successful to probe the structural and dynamic properties of supported lipid bilayers but one of the most popular experimental approach, is the use of the Quartz Crystal Microbalance. ${ }^{39}$ The Quartz Crystal Microbalance represents a key technique in the study of SLB systems. Its role has been pivotal since the first studies on vesicle rupture and fusion on surfaces appeared, thanks to the fact that QCM measurements offer a straightforward way to follow in real time the sequence of events leading to the formation of SLB. ${ }^{40}$ Modern QCM instrumentation measures simultaneously the resonant frequency shift $(\Delta f)$ and the change in energy dissipation $(\Delta D)$ of a gold coated quartz crystal at the fundamental resonance frequency as well as for the higher overtones, as a substance adsorbs on the sensor surface. If the mass adsorption is evenly distributed, rigidly attached to the crystal, and small if compared to the mass of the crystal, then $\Delta f$, the measured frequency shift, is linearly proportional to the mass density, $\Delta m / A$, of the deposited film according to the Sauerbrey equation. ${ }^{41}$ Although this relationship can be extended to a quasi-rigid film, for thicker or spongy films the resonance frequency is affected not only by the mass attached to the surface but also by the viscoelastic properties of the adsorbed film. In this case, global fitting of $\Delta f$ and $\Delta D$ data collection is generally performed using Voigt-based models, ${ }^{41}$ which were proved to be well suited for layers of organic molecular or higher aggregates as phospholipid vesicles. ${ }^{40,42}$ These modelling procedures provide the wet mass structure in terms of average thickness and surface density, together with the viscoelastic parameters of the adsorbed layer. Since QCM is a microgravimetric method, the results are always overestimated due to the contribution of the adsorbed water in the resulting adsorbed mass. Such effect can be neglected as long as the focus is on the comparison between the thickness of the adsorbed layers from QCM data for SLB and that obtained from DLS experiments on vesicles.

If water-free data are strictly required it may be convenient to combine a spectroscopic surface technique as ellipsometry or surface plasmon resonance to QCM investigations. Recent combined QCM and ellipsometry results from our group on SLB formation from extremely diluted liposome dispersions converged in a two-step mechanism for SLB build-up at the surface.

As shown in Fig. 5, QCM thickness is consistently higher that its ellipsometric counterpart, although both sets of measurements show a first arrest, likely corresponding to monolayer coverage of the surface. For this reason, ellipsometric data should not be compared with DLS data on added hydrodynamic thicknesses, but rather with SAXS and SANS data on the same systems, provided the correct scattering length density profile is considered. Furthermore, thanks to the possibility to obtain a surface mapping of the elliposmetric thickness of the adsorbed layer in situ, imaging ellipsometry will be increasingly used in SLB-related investigations in the near future. In spite of the fact that the lateral resolution is limited to $1 \mu \mathrm{m}$, differences in thickness of $1 \mathrm{~nm}$ can be conveniently appreciated with this method. Also mechanical probing techniques provide a high resolution mapping of the thickness of the bilayer as well as on the presence of defects, vacancies, phase separated domains or intact trapped vesicles but, differently from Atomic Force Microscopy, imaging ellipsometry can be easily operated as the SLB is progressively forming on the surface. Moreover the method is safely nondestructive and does not require a direct physical interaction

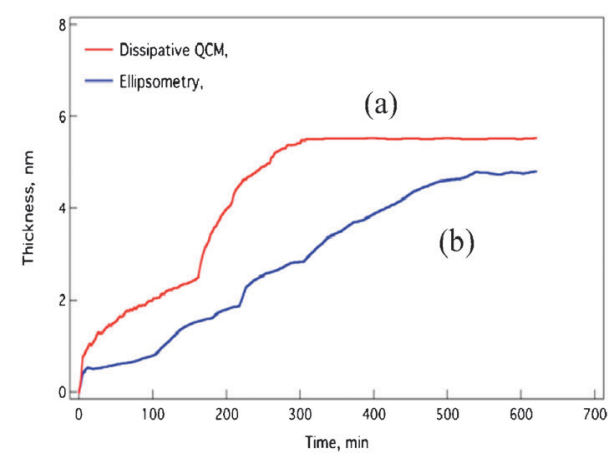

Fig. 5 QCM (a) and ellipsometric (b) thickness after addition POPC liposomes to the hydrophilic solid support. 
with the sample that may lead to unwanted disruption of the SLB layer.

Investigation on functionalized phospholipid bilayer benefits also of from fluorescent-based methods, i.e. fluorescent probing, fluorescence energy transfer ${ }^{43}$ and total internal reflection fluorescence microscopy. ${ }^{44}$ While conventional fluorescence experiments can be successfully developed for liposomes in solution, the same is not true for SLB systems where the scarce overall signal hinders sufficient data collection. This drawback can be overcome with the use of fluorescence imaging techniques: although a SLB is a only 5-nm-thick membrane, it can be easily observed with modern Confocal Laser Scanning Microscopes (CLSM). In the past few years, CLSM has become an invaluable tool for a wide range of investigations in biological and medical sciences for imaging of thin optical sections, but pairing of this sound methodology with supported bilayers is still in its infancy. As for other fluorescent techniques, CLSM requires the incorporation of a small number of dye-conjugated lipid molecules into the fabricated SLB. When excited by the proper wavelength, the dye provides visible fluorescence that can be collected and imaged in the $x y$ plane and along the $z$-axis. The advantages of CLSM are manifold: spanning from in-focus 3D imaging to spatial- and time-resolved spectroscopy in situ. Whereas parallel AFM and QCM measurements have been reported in the past, ${ }^{28} \mathrm{QCM} / \mathrm{CLSM}$ and CLSM/AFM studies on supported membranes are less common although comparison between AFM and CLSM measurements provide a definite proof on the reliability of probe-dependent measurements. In fact, the addition of a fluorescent dye is generally believed not to perturb the system, a statement that is often proved false. Equivalence of CLSM images and AFM images of the analogue probe-free SLB ensures that further fluorescence applications, such as FRET or FRAP, can be safely performed both on SLV and on liposomes with the same composition. In our group, we recently applied the CLSM technique to the study of SLB decorated with cholesterol-tagged DNA (see Fig. 6): fluorescence modification was achieved using a fluorescein-labelled single strand oligonucleotide $\left(\mathrm{ON}_{2} \mathrm{FAM}\right)$ with its complementary sequence $\left(\mathrm{SC}-\mathrm{ON}_{1}\right)$ (see Fig. 7) anchored to the SLB layer. SLB were formed on mica from POPC liposome and the chol-tagged double strand was successively added to the system. An example is shown in Fig. 6 that reports the CLSM mapping of the functionalized SLB formed on the mica surface compared to AFM images of the same sample together with the emission spectra of the fluorescent label recorded in different SLB regions.

Both imaging methods evidence the formation of a bilayer of similar morphology and comparable thickness, although lower spatial resolutions are achieved with the CLSM technique. Nevertheless, CLSM largely compensates this drawback providing two additional features: in the first place it permits to assess that the fluorescent probe, and hence the DNA double strand, is evenly anchored to the surface of the phospholipid bilayer.

More importantly, spatially-resolved photophysical characterization of selected surface regions is readily accomplished as shown in Fig. 6c where the characteristic emission spectra of fluorescein in different regions of the SLB surface spectra are
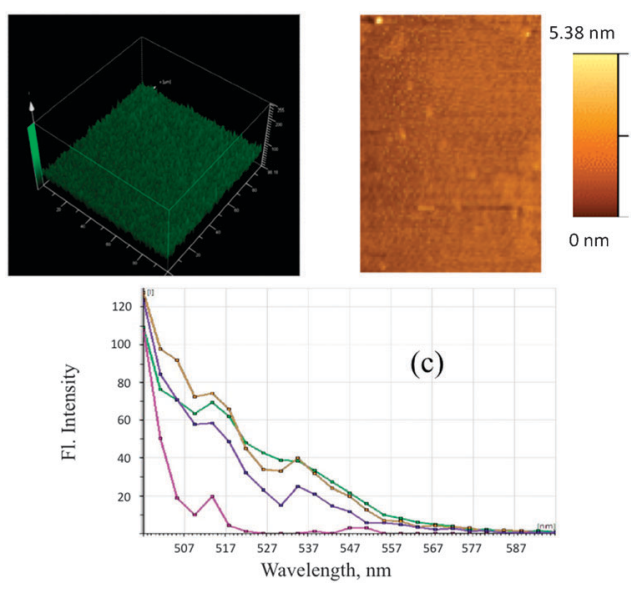

Fig. 6 (a) CLSM tridimensional imaging of $\mathrm{SC}-\mathrm{ON}_{1} / \mathrm{ON}_{2} \mathrm{FAM}$ decorated SLB; (b) AFM images of the same sample; (c) spatially resolved spectra for different bilayer domains exhibiting the characteristic signature of fluorescein.

(A)
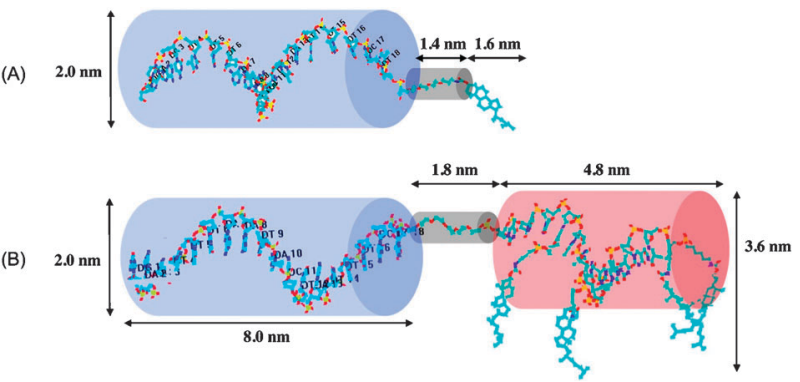

Fig. 7 Chemical structure of SC-ON 1 and $\mathrm{MC}-\mathrm{ON}_{1}$, hydrophobically tagged oligonucleotides, inserted as guests in liposomes and SLB in a parallel study.

shown. Interestingly, the contribution of the fluorescein dianion and monoanion species to the emission spectrum, which is a feature strictly correlated to the local charge density sensed by the probe, resulted to be very similar to that previously found with conventional fluorescence spectroscopy for liposome dispersions with the same composition.

\section{Liposomes and SLB: some important differences}

Research papers that explicitly address the comparison of structural and dynamical properties of planar and curved bilayers are not very common. The parallel analysis of bilayer systems with an identical composition, an identical local but different long-range orders, is the main focus of this feature article. In our review of the literature on the subject, we should underline that some obvious physical differences, which might not represent a concern when studying simple bilayer systems, become critical in the comparison when functional molecules are inserted into bilayer matrices.

In this paragraph we will summarize the main general aspects concerning this issue.

We have already mentioned possible effects on the curvature that can be significant for small liposomes (radius $<50 \mathrm{~nm}$ ), considering the most common lipid constituents. 
Several studies in the literature have addressed the effect of curvature on the local structure of the bilayer, reporting seemingly contradictory results, from a bilayer thinning as the liposomal radius increases, to asymmetries in the bilayer profile. Recently, a careful SANS analysis, combined with high-resolution SAXS has revealed no effect of curvature on lipid bilayer structure for zwitterionic vesicles in the range of diameters $60-180 \mathrm{~nm}$, while for anionic lipids, an asymmetry between the outer and the inner leaflet was detected for vesicles with $100 \mathrm{~nm}$ diameter, possibly due to coupling with counterions distribution. ${ }^{45}$

One important structural parameter is the total available interface for inclusion in the hydrophobic compartment and for interaction with hydrophilic molecules, either bound to the bilayer or in solution. For preformed liposomes this is given by the total area of the external leaflet that can be easily evaluated, knowing the lipid concentration, the hydrodynamic radius and the bilayer thickness. Usually this area is larger than one half of the total area, especially for SUV, thanks to the higher number of lipid molecules occupying the external leaflet and is given by:

$$
\frac{S}{V}=N_{\mathrm{A}}[\text { lipid }] A_{\text {lipid }} \frac{R^{2}}{R^{2}+(R-t)^{2}}
$$

where $S / V$ is the interfacial area per unit volume, $N_{\mathrm{A}}$ is the Avogadro number, $A_{\text {lipid }}$ is the lipid cross-section, $R$ the vesicle radius and $t$ the bilayer thickness. The bilayer thickness and the area/lipid molecule are reported in the literature for the most common lipids, while the hydrodynamic radii and their polydispersity should be determined for any given liposomal batch.

Obviously, the interfacial area for a SLB is one half of the total interfacial one. The other fundamental difference concerns the connectivity of the two bilayered systems. In one single fluid monophasic SLB, the hydrophobic/amphiphilic guests (i.e. a molecule or a biomolecule partially or totally embedded in the bilayer), if free to diffuse laterally, are virtually connected with all the remaining ones.

The distribution of guest molecules among liposomal hosts follows a Poisson distribution, which approximates a Gaussian distribution only for relatively high occupancy numbers, i.e. guest molecules per liposomes. The picture is utterly complicated by the possible polydispersity, which induces fluctuations between the occupancy numbers, even for relatively high average numbers of hosts/liposomes. ${ }^{46}$

The probability of finding a guest " $i$ " per liposomal hosts is given by

$$
P_{i}=\frac{N^{i}}{i !} \exp (-N)
$$

with $N$ the occupancy number, $n_{\text {guest }} / n_{\text {vesicle }}$ and $n_{\text {vesicle }}$, the total number of vesicles per unit volume, is given by

$$
n_{\text {vesicle }}=\int \frac{[\text { lipid }] N_{\mathrm{A}} A_{\text {lipid }}}{8 \pi R^{2}} \frac{R^{2} P(R) \mathrm{d} R}{\int R^{2} P(R) \mathrm{d} R}=\frac{[\text { lipid }] N_{\mathrm{A}} A_{\text {lipid }}}{8 \pi \mu_{R, 2}}
$$

where $P(R) \mathrm{d} R$ is the probability of finding liposomes having radii comprised between $R$ and $R+\mathrm{d} R, \mu_{R, 2}=\sigma_{R}{ }^{2}+\langle R\rangle^{2}$, the second moment of the size distribution, accessible from a
DLS experiment (vide infra), and with the simplified assumption of even distribution of the lipids among the two leaflets.

All these factors can be relevant for particular cases, such as low hydrodynamic radius and high polydispersity and should be properly considered when comparing different bilayer systems.

Another aspect is connected to experimental consideration, and concerns the total working concentrations. For instance, we have reported that for a millimolar lipid concentration, a liposomal solution consisting of $35 \mathrm{~nm}$ radius low-polydisperse vesicles provides an available interface having an area more than six orders of magnitude higher than the area of a SLB coating a QCM crystal $\left(8 \times 10^{13} \mathrm{~nm}^{2}\right.$ versus $\left.3 \times 10^{20} \mathrm{~nm}^{2}\right)$. This requires that, to realize a given lipid/guest ratio, the guest concentration in solution should be correspondingly shifted. For amphiphilic guests, this concentration difference can result in competing aggregation equilibria, so that partition involves actually three species, i.e. the monomer in solution, in the aggregate and in the vesicle-bound state.

Last but not least, we should consider that, when adding amphiphilic or hydrophilic molecules (such as proteins displaying peripheral binding through Coulomb interactions with charged membranes) to preformed liposomal dispersions, an equilibrium partition between lipid vesicles and the surrounding medium is established. Gel filtration can be performed to exclude guest molecules not bound - or enclosed in-liposomes. For SLB, this operation is usually automatic, i.e. the binding event is followed by rinsing treatments in most experimental protocols; therefore the binding degree is not ruled by thermal equilibrium, but is rather a metastable state, where desorption events can be observed. This results in minimal differences for high binding constants, but can be relevant in some cases.

One of the major drawbacks of supported bilayers is certainly the close proximity of the inner layer with the solid surface: SLB are generally separated from the solid support by only $1-2 \mathrm{~nm}$ thick layer of water. This feature limits severely the possibility to incorporate integral membrane proteins, which can denature when in contact with the substrate surface. This issue has been extensively elaborated in recent reviews ${ }^{20,24}$ that summarized the results obtained both spreading vesicles containing reconstituted integral proteins and incorporating 'anchor' molecules to the SLB followed by coupling of engineered proteins to those anchors. Supported membranes containing reconstituted proteins have already provided information about several important biological processes but the studies also evidenced that there is not yet a completely satisfactory supported membrane system for the inclusion of transmembrane proteins with both large extracellular and intracellular peripheral domains.

Such limitation is less strict in liposome system where an additional bonus is offered by possibility to vary the liposome dimension and hence the curvature radius and the size of inner water pool.

Contrary to liposome systems, SLB may easily undergo phase segregation and delamination when interaction with the solid surface predominates. In an effort to overcome these limitations different groups proposed lipopolymer protection linking a close-packed polymer layer in tight brush conformation. ${ }^{47}$ 
The polymer is transferred on top of the SLB surface by the LB technique, this modification increases relevantly the bending elastic modulus of the bilayer raising the barrier to delamination. In addition, the presence of the hydrophilic protein layer inhibits lipid reorientation after air exposure resulting in an air-stable supported bilayer.

Each of the above aspects are general and contribute to the fundamental expertise of bilayer science. There are however a number of more specific issues that arise when addressing particular problems. These will be exemplified through the illustration of three case studies.

In the first one, the comparison between supported lipid bilayers and vesicles in solution is explicitly addressed and the structural and functional insights on both systems are discussed within a common platform to get an integrated picture. In the second and third example, we will focus on two popular topics in the research area of lipid bilayers: synthetic models for lipid rafts and compositionally asymmetric bilayers. Generally, in these latter cases reports are dealing with SLB or vesicles. The inclusion of such examples in the framework of this Perspective is aimed at underlining the benefits that an integrated approach, comprising the analysis of both systems, would enable in terms of fundamental knowledge and biomimetic purposes.

\section{Case studies and perspectives}

\subsection{Incorporation of lipophilic oligonucleotides in lipid bilayers}

Lipid self-assembly is one of the bottom-up strategies conceived by Nature to produce matrices with (multi)functional control at the nanometre length scale. This approach can be borrowed by soft matter-based nanotechnology, and further integrated through incorporation of complex structural motifs in the amphiphilic building blocks, either synthetic or bio-inspired ${ }^{48}$ as in the case of covalent conjugation of nucleosides ${ }^{49}$ or oligonucleotides $^{50}$ to amphiphilic assemblers. Through this functionalization, one obtains oligonucleotides building blocks with tailored properties for controlled self-assembly, which can be anchored to soft surfaces, such as planar or curved phospholipid bilayers. ${ }^{51}$ The ordered assembly of lipid hosts provides a structural skeleton, enhanced local concentration, and/or immobilization and/or favorable orientations for the guest oligonucleotides. The final goal is to further extend the range of application of DNA-directed construction of nanomaterials, which exploits the structural fidelity, and coupling specificity, enabling further hierarchical aggregation in functional arrays of nanounits. Our group has recently studied the incorporation on a singly substituted derivative $\left(\mathrm{SC}-\mathrm{ON}_{1}\right)$ and a multicholesterol $\left(\mathrm{MC}-\mathrm{ON}_{1}\right)$ derivative, where the cholesteryl units are inserted at the desired positions along a non-coupling T-sequence, whose structure is reported in Fig. 7, in phospholipid vesicles and supported lipid bilayers and their hybridization with a complementary strand.

The results have been interpreted in terms of average distance between non covalent grafting sites onto the membrane as the independent parameter, i.e. number of hydrophobic oligonucleotides per interfacial area unit. All the concepts outlined in the previous paragraph for a correct comparison of the two systems have been applied to evaluate the interfacial area. ${ }^{32}$

The hydrophobically tagged oligonucleotides have been added, at increasing concentrations, to a highly monodisperse liposomal solution and to SLB.

While DLS measures a decrease in diffusion coefficients, ascribable to an added hydrodynamic thickness, due to the build-up of an oligonucleotide layer surrounding the vesicle, QCM measures the mass increase due to bilayer insertion for the same guest/lipid ratios. Fig. 8 reports the trends of these two quantities for the single cholesteryl derivative, as the average grafting density is varied. ${ }^{32}$ It should be stressed that DLS becomes in this case a very sensitive technique, since the added hydrodynamic thickness amounts to a considerable volume increase of the scattering objects, allowing to overcome the size detection limit of DLS.

Interestingly, in both cases we have a saturation threshold that occurs at the same area/guest molecule ( 80 lipid molecules per guest molecules), notwithstanding the different overall concentration of the added oligonucleotides.

Moreover, the hydrophilic layer thickness at the saturation threshold (about $60 \AA$ A) determined through QCM well-matches the added hydrodynamic thickness measured by light scattering.

These results unequivocally support the structural similarities between the two models and allow dynamical transfer of information between free-standing and supported single bilayers.

An example of such possible interplay is provided by a parallel Linear Dichroism study on these oligonucleotide-decorated
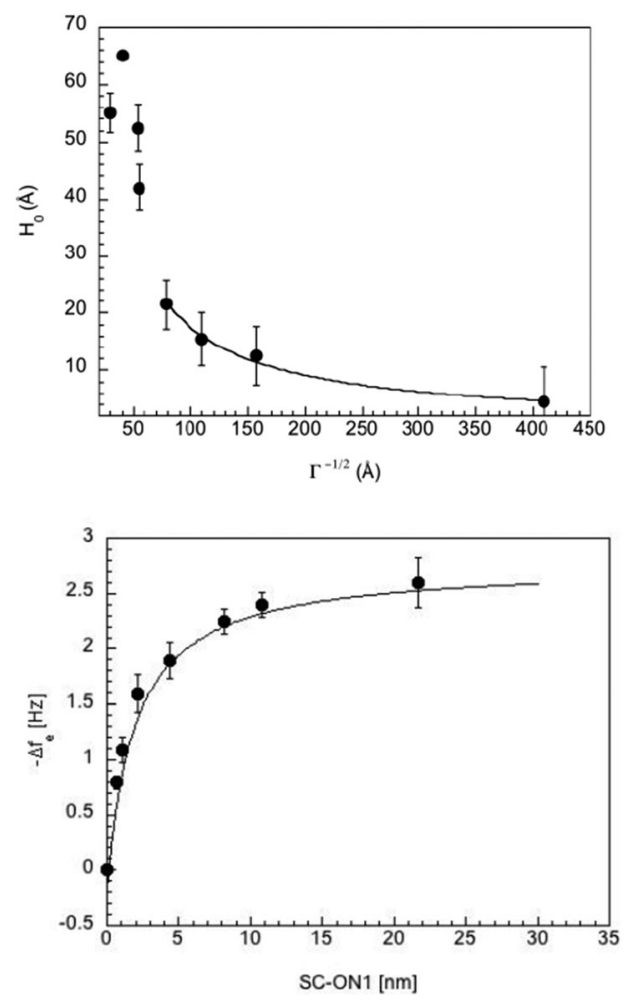

Fig. 8 Upper panel: Added hydrodynamic thickness around a lipid vesicles, as a function of the average distance between non-covalent grafting sites. Lower panel: Frequency shift observed by QCM as a function of the overall concentration of SC-ON $\mathrm{N}_{1}$. 
ULV under shear-flow, ${ }^{32}$ which indicate that this increase in hydrodynamic thickness is connected to orientational variations of the oligonucleotide chain with respect to the bilayer director as its non-covalent grafting density is increased. The same mechanism can be considered to be operating on planar supported bilayers.

Moreover stopped-flow experiments on ULV can probe the coupling kinetics between the bilayer-embedded oligonucleotides and the complementary oligonucleotide in solution, i.e. the complementary strand without any hydrophobic label.

A remarkable result was the fact that above a critical grafting density, connected to the lipophilic oligonucleotide excluded area, the membrane-assisted hybridization kinetics are slowed down relative to strand pairing in solution. Conversely, when the average oligonucleotide density on the liposomal surface is low, coupling is faster than in bulk medium. This result can be correlated to the same reaction occurring in planar bilayers, which is relevant in the field of DNA microarrays, whose development for analytical purposes could greatly benefit from dynamic studies. Clearly, a high-throughput technique as stopped-flow spectroscopy in solution, performed without need for any molecular probe, as fluorescence dyes, warrants reliable and sound results for any modelling.

As already noticed, one often neglected aspect is the possible occurrence of aggregational equilibria for amphiphilic hosts. In this respect, one illustrative example is provided by the comparison of the previous derivative with another multicholesterololigonucleotide (MC-ON 1 , Fig. 7, bottom) differing in the architecture and hydrophobicity of the lipophilic moiety. ${ }^{40,50}$ In this study the comparison between free-standing and supported single bilayers is explicitly taken into account, likewise the selfaggregation properties in solution of the guest molecules.

Fig. 9 reports the adsorption isotherm in terms of hydrodynamic thicknesses for ULV, together with the thickness increase resulting from the normalized frequency shift of the resonant frequency of QCM crystal.

The adsorption pattern on the planar bilayer supports the picture of a three-regime mechanism, where, beyond monolayer saturation, the presence of free cholesterol units protruding in solution, promotes aggregation near the interface. The adsorption mechanism, beyond the unimers domain, was rationalized applying a Tetris-like compaction model to the QCM data.

With respect to DLS measurements, the lipid/guest ratios explored are those corresponding to the monolayer saturation regime, i.e. the first of the three domains explored by QCM, but the bulk concentration range is shifted more than one order of magnitude. A parallel investigation on the self-aggregation of these amphiphilic oligonucleotides, reveal a peculiar aggregation pattern, whose threshold is for $\mathrm{MC}-\mathrm{ON}_{1}$ at 0.2 micromolar. Therefore, in DLS experiments, depending on the bulk concentration, either unimers or aggregates are in equilibrium with vesicles, while for QCM experiments, only unimers are expected in bulk solution.

The total phospholipid surface is different, not only quantitatively but also from a dynamical point of view. For liposomes, it consists of disconnected domains (the vesicles themselves), while for SLB there is a continuous, macroscopically extended bilayer domain.
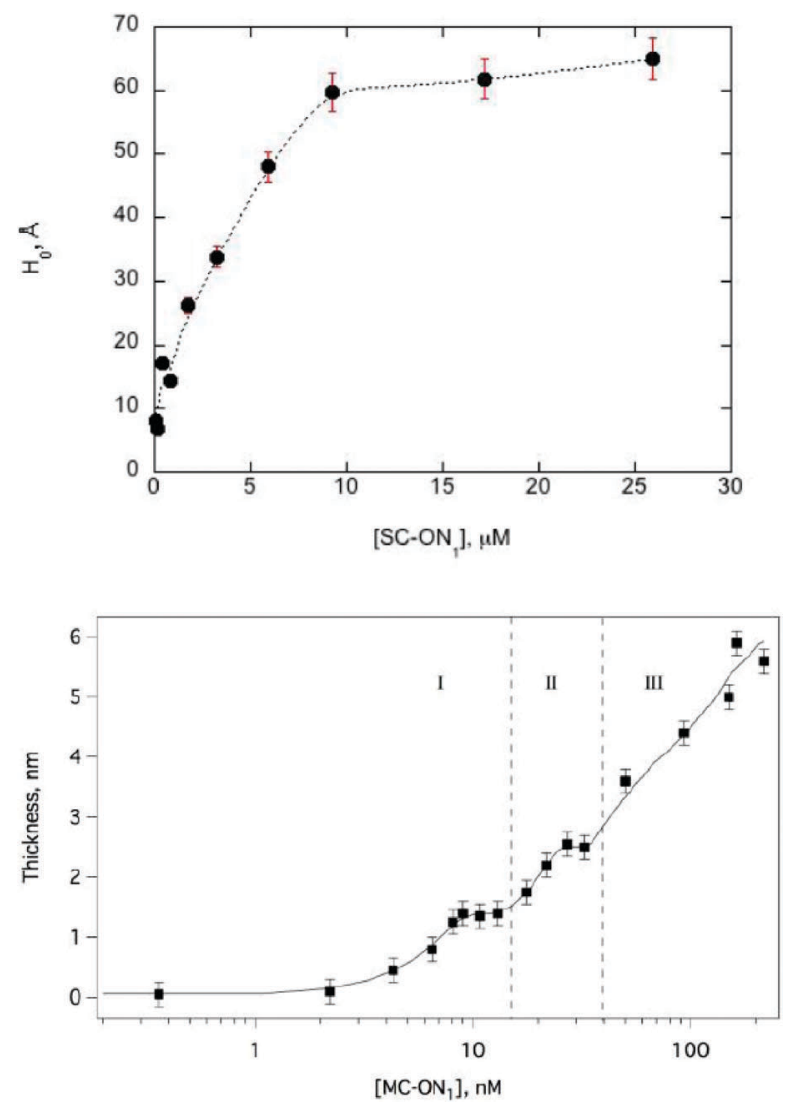

Fig. 9 Upper panel: Hydrodynamic added thickness of ULV as the guest concentration is increased. Bottom panel: QCM thickness as a function of $\mathrm{MC}-\mathrm{ON}_{1}$ concentration. Note the different concentration ranges.

Therefore, any cooperative process may lead to different products also for an identical phospholipid/amphiphilic DNA ratio: this applies both to lateral interactions in the membrane and to membrane-induced processes at the lipid/aqueous interface.

\subsection{Phase separated domains in lipid bilayers as mimics of membrane rafts}

Lipid rafts are dynamic membrane domains, with a typical diameter of around $10 \mathrm{~nm}$, enriched in sphingolipids and cholesterol, which form detergent-resistant bodies within membranes. ${ }^{52}$ It is commonly accepted that rafts consist of liquid-ordered phase domains (Lo). The acyl chains of lipids are in all-trans configuration, ordered as in the gel phase but with high lateral mobility within the membrane plane. Several receptor proteins interact preferentially with lipid rafts, which have a key role in signal transduction.

The fact that such microdomains can be observed even in thermally equilibrated simple model systems, ${ }^{53}$ for some given compositions, has prompted the investigation of their structural and dynamic properties, excluding the structural and functional complexity of the biological archetypes.

In addition, lipid rafts are implicated in processes such as endocytosis, exocytosis, and vesicular trafficking (transport of vesicles across the cell) and are therefore associated with regions of enhanced membrane curvature. Localized changes 
to membrane curvature in cells are essential for inter- and intracellular communication. In model systems, externally induced curvature changes have been observed to drive lateral organization. ${ }^{54}$

From what above said, it is clear that membrane curvature and lateral phase separation are intimately intertwined and the understanding their interplay can be one of the future challenges in the field. More specifically the size of the phase separated domains, and its connection with the membrane curvature, can be a critical factor, in particular when the function of lipid rafts is considered.

One of the recent reasons of interest towards lipid rafts is the increasing experimental evidence of the implication of these domains in pathologically relevant phenomena, which involve the spatiotemporal regulated distribution of membraneassociated proteins, in terms of accumulation and segregation and eventually misfolding and aggregation. ${ }^{55}$

In this hypothesis the spatial extension, the coalescence dynamics and the connectivity of lipid domains are clearly critical factors, whose consideration must be necessarily taken into account. It should be stressed that rafts in the cells have typical sizes of some tens of nanometres, i.e. one or two order of magnitudes smaller than the most popular synthethic mimics, i.e. micron-sized lateral domains in Giant Unilamellar Vesicles, usually imaged thanks to the different affinity of fluorescent probes for the liquid ordered and the disordered phase. ${ }^{56}$ Some FRET studies have, for instance, suggested phase separated domains in ULV for lipid compositions where fluorescence microscopy did not reveal any lateral heterogeneities for GUVs. ${ }^{57}$

It is therefore clear that inhomogeneities occur in two length scales, ranging from micron-sized domains to domains of diameter smaller than $10 \mathrm{~nm}$.

The techniques of investigation of liposomes are typically ensemble-averaged in solution, which is of course an advantage with respect to microscopy, where preparation artifacts or the necessarily low number of individual objects observed can lead to un-representative results. On the other side, colloidal objects undergo Brownian motions and collisions in solution: in the case of functional liposomes, mutual interactions mediated by the function (i.e. aggregation mediated by a protein partially inserted in the bilayer) might be very difficult to decouple from genuine effects.

These considerations nicely illustrate the complementarity of the lipid scaffolds of interest in the perspective of using synthetic lipid rafts to elucidate their role in pathologically relevant phenomena at a molecular level. Convincing and reliable results will necessarily involve both liposomal systems (size of the liquid ordered domains) and SLB (size of the liquid disordered matrix and guest/triggered coalescence phenomena) observed either with fluorescence microscopy or with higher resolution techniques (i.e. AFM, or neutron reflectivity).

For liposomes, neutron contrast variation obtained through the appropriate use of selective deuteration, enables the qualitative and quantitative structural characterization of liquid ordered rafts in ULV through SANS $^{58}$ in the underlying assumption that lipid chain deuteration of one component does not affect phase behaviour for the studied compositions.
One of the central results of these SANS studies is the fact that membrane curvature has a significant role for the miscibility of the various lipid components and that increased membrane curvature induces lipid demixing. ${ }^{59}$

While this result is encouraging, as it confirms that local curvature can modulate lipid composition, as in cell membranes, it poses some fundamental questions as to which is the most reliable model for lateral inhomogeneities. Of course there is no clear answer to this question, and presumably in the next future, both models should be taken in consideration and critically compared.

The formation of a single SLB on a solid support from the same ULV where nanodomains are observed and the coalescence or disappearance of phase domains, followed with GISANS or GISAXS techniques, possibly coupled with studies on reflectometry, would represent an experiment of sure interest.

The disappearance of such domains would confirm the role of the curvature, while their persistence would in turn naturally introduce novel fundamental questions to be addressed. One important point concerns the compositional asymmetry of the outer and the inner leaflet of the bilayer. We should stress that in the exofacial leaf of the plasma membranes, lipids that in model systems give rise to phase separated domains (sphingolipids, glycophospholipids, cholesterol) are usually overexpressed, while lipids belonging to the cytosolic leaf (phosphatidylserines and phosphatidylethanolamines, together with glycophospholipids) when reconstituted in model membranes do not show lateral segregation domains. ${ }^{53}$ The interleaflet coupling of phase separation, which is an activated process in Nature, provides a pathway for signal transduction between the extracellular and the cytoplasmic compartment. For ULV, the coupling between leaflets is difficult to prove, and there is no obvious direct experiment to propose. Actually, for multilamellar systems (either planar or curved), coupling between adjacent layers has been observed for raft structures. ${ }^{60}$ Therefore trans-monolayer coupling of rafts in the presence of nanosized lateral inhomogenities appears reasonable. Since phase-separated domains are usually observed in ternary lipid mixtures, including cholesterol, it is very likely that their quenching upon membrane curvature reduction would occur with lipid redistribution between the two leaflets. The experimental proof of such an event would undoubtedly provide improved understanding of biochemical processes at a molecular level.

On the other hand, supported lipid bilayers have been extensively used in understanding the fundamental properties of heterogeneity in biological membranes and a substantial literature has been published on studies of lipid domains in SLB. Many authors have investigated lipid mixtures with coexisting gel and liquid-crystalline phases by atomic force (AFM), epifluorescence, and near-field fluorescence microscopy (NSOM). Several of these studies demonstrated the coexistence of ordered and disordered phases for a variety of different lipid compositions in SLB. However, there are several discrepancies between the structural organization of proposed rafts in biological membranes and phase-separated domains in model membrane systems. For example, in nearly all investigations on supported lipid bilayers formed through 
vesicle fusion, lipid domains are transmembrane symmetrically distributed; very little work has been done to study transmembrane asymmetric distributions in these systems. Remarkably, Longo and coworkers ${ }^{61}$ recently described three different states of transmembrane symmetry in phase-separated supported lipid bilayers formed by vesicle fusion by a combined atomic force microscopy and fluorescence microscopy study. The authors observed that if the leaflets differ in the gel-phase area fraction, the smaller domains in one leaflet are aligned with the larger domains in the other leaflet and the system is dynamic, involving in the erosion of the smaller domains in one leaflet by lipid flip-flop processes. At the same time the large domains in the other leaflet grow resulting in a complete compositional asymmetry. On the contrary, if both leaflets have identical area fraction of gel-phase, gel-phase domains are in registry and static in comparison to the first state. ${ }^{61}$ Recently, Sutherland and coworkers ${ }^{62}$ studied SLB formed through rupture of ULV with lipid composition chosen to correspond to $\mathrm{L}_{\mathrm{d}}, \mathrm{L}_{\mathrm{o}}$ phase domains in established phase diagrams of 1-palmitoyl2-oleoyl-sn-glycero-3-phosphocholine (POPC), N-palmitoylD-erythro-sphingosylphosphorylcholine (PSM) and cholesterol. QCM results demonstrated that inclusion of PSM and cholesterol into POPC vesicles significantly impaired the vesicle rupture process. When increasing the cholesterol content the vesicles either formed SLB containing more defects or resisted the rupture process forming supported vesicular layers. Also, the presence of raft-like domains in SLB systems formed by ULV fusion was imaged by AFM by Kane and coworkers exclusively after thermal annealing treatment of the sample. ${ }^{63}$ Conversely, Rädler characterized by synchrotron X-ray reflectivity the structure of liquid-ordered and liquid-disordered SLB formed upon insertion of the membrane receptor GM1 on preformed SLB; ${ }^{64}$ asymmetric structural changes across lipid bilayer leaflets were shown to be induced by the incorporation of GM1 into the outer leaflet layers.

\subsection{Asymmetric bilayers: towards the complexity of biological archetypes}

From what we have stressed in the previous paragraph, the control over differences in lipid composition appears crucial to bring the experimental approaches on cell membranes and on model counterparts closer together and achieve more quantitative agreement, especially in the field of structure and function of lipid rafts.

Since the first studies on Supported Lipid Bilayers (SLB) appeared two decades ago, ${ }^{17}$ an on-going debate on the biomimetic fidelity of SLB has developed, especially on aspects such as mobility and asymmetry of the bilayer. Tamm et al. recently discussed the relevance of SLB in chemical biology stressing how Supported Lipid Bilayers have evolved into reliable membrane models. ${ }^{65}$ Supported Lipid Bilayers are intrinsically asymmetric, because of the interaction with the solid support. Moreover preparation techniques can easily yield bilayers where each monolayer has a different composition.

The interleaflet distribution of the phospholipids is commonly assumed to be symmetric, although many works suggest that an asymmetrical lipid distribution of lipids in SLB may be more prominent than commonly appreciated. ${ }^{47}$ Richter et al. ${ }^{66}$ demonstrated that calcium-mediated "specific" interaction between DOPS and mica resulted in the formation of asymmetrical SLB proposing that asymmetry generates in the intermediate step on vesicle rupturing on the surface via a phospholipid flip-flop across the edge of the thinning liposome at the surface. ${ }^{66}$

Asymmetric SLB were prepared in our group using ds-DNA decorated liposomes as fusing media as shown in the Fig. 10.

Confocal Laser Scanning Microscopy images reported in Fig. 11 were collected for SLB formed with this procedure as a function of contact time between the liposomal dispersion and the surface.

It is evident from the CLSM images that the presumably asymmetrical SLB formed when the hydrophobic anchoring is present only in the outer surface of liposomes are not stable and irreversibly tend to phase separate in large domains enriched with the double strand modification.

Instability may be due to a larger fraction of defects combined with a non-negligible flip-flop probability between the outer and the inner phospholipid layer, such effect is expected to be surface-specific and could be reduced or suppressed with the addition of a cushion layer.

The above approach has often been indicated as beneficial to overcome limitations in SLB construction protocols developed so far, but our results as well as other literature reports indicate that the structural composition of functionalized liposome surfaces cannot be replicated on SLB systems by direct liposome fusion on the surface. ${ }^{27}$

Other strategies may be more fruitful to develop stable and reliable asymmetrical SLB: these methods have been detailed in recent reports ${ }^{27}$ and include the deposition of a LB layer on a hydrophilic solid support followed by adsorption and disruption of vesicle with a different phospholipid composition compared to the first LB layer (LB/VF method). This procedure, although simple, results in asymmetrical bilayers whose fluidity properties closely match those of the native LB layers. Even larger stiffness is determined when the first LB layer is replaced by a chemically bound self-assembled-monolayer. The resulting SLB may differ significantly from the lipid bilayer in liposome systems and more importantly from natural membranes, nevertheless for specific applications, such as the fabrication of nano-sensor arrays or patterning devices, such rigidity may enhance the SLB performance. ${ }^{67}$

The above strategies are necessarily hindered in the case of liposome solutions: currently, the conventional preparation protocols of ULV do not provide transleaflet controlled compositional difference. Recently, an increasing awareness

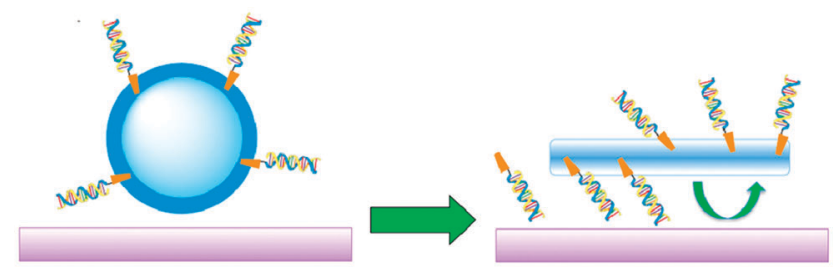

Fig. 10 Formation of $\mathrm{ON}_{2} \mathrm{FAM} / \mathrm{SC}-\mathrm{ON}_{1}$ anchored SLB through direct spreading of $\mathrm{ON}_{2} \mathrm{FAM} / \mathrm{SC}-\mathrm{ON}_{1}$-liposomes on mica surfaces. 

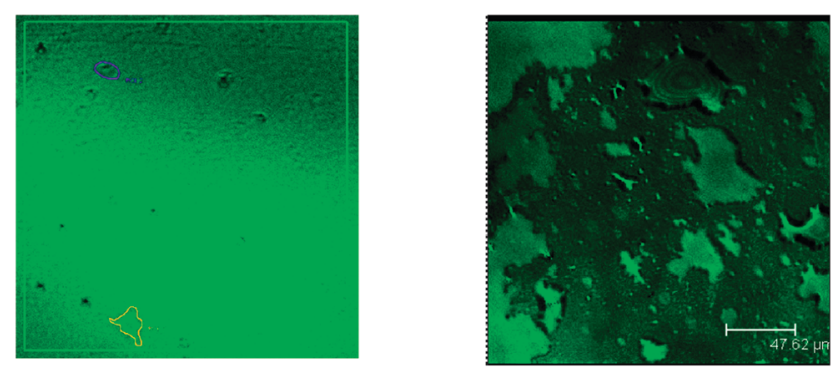

Fig. 11 SLB obtained from of $\mathrm{ON}_{2} \mathrm{FAM} / \mathrm{SC}-\mathrm{ON}_{1}$ decorated liposomes as a function of contact time, $[\mathrm{ON} 1]=[\mathrm{ON} 2]=4.15 \mathrm{mM}$. (a) $t=24 \mathrm{~h}$, (b) $t=48 \mathrm{~h}$ (scale bar $=50 \mu \mathrm{m})$.

of the importance of the topic has concurred to direct scientific efforts towards the aim of providing solid methods to overcome this limitation. We should mention that, even in the case of one-component vesicles, there is no guarantee that leaflets are physically equivalent, depending on the curvature, as demonstrated recently for charged vesicles. The picture is further complicated for binary and ternary lipid blends, where membrane asymmetry is very likely to occur, also in compositional terms. ${ }^{68}$

From the previous considerations, the search for reliable, medium/high-throughput methods for the controlled production of asymmetric vesicles is surely one of the future challenges in the field of lipid bilayers, both as building-blocks for hierarchically assembled nanoscale constructs and as reliable platforms for biomembrane mimics. However the purpose of increasing complexity levels should not jeopardize the structural and dynamical control, which represents the key asset of model systems.

Partial asymmetry has been reached in the past thanks to chemical methods, such as $\mathrm{pH}$ changes or osmotic pressure alterations, which act on the outer layer. ${ }^{69}$ Recently, some authors have proposed the use of a physical method where a lipid without emulsion in dodecane is layered on top of a twophase system consisting of a denser oil phase and an aqueous solution, separated by an interface where the outer layer lipid is assembled. After centrifugation the asymmetric vesicles are recovered in the aqueous phase. ${ }^{70}$ The principal current limit of this method and of some recent developments, is the sizerange, which is in the GUV domain, and therefore precludes control of curvature, which is closely connected with raft formation. A novel chemical method, where sphingomyelin and cholesterol are selectively introduced in the outer leaflet of preformed ULV of the desired size, has been recently proposed $^{71}$ and its future developments can be of great help for research in this field.

The formation of SLB from asymmetric ULV will be undoubtedly a very fertile area of research and will considerably improve our current understanding either on the formation of single supported bilayers and on the effect of curvature on asymmetry.

\section{Conclusions and outlook}

The high level of specificity and complexity of experimental techniques for the investigation of lipid bilayers have in some case resulted in highly sectorial, though excellent, scientific expertise.

A critical evaluation of the panorama of current trends in liposome-focused systems and supported bilayer scenarios unquestionably revealed that cross-fertilization between the two investigation fields is highly beneficial to both scientific communities.

Spatially resolved and time-resolved understanding of surface and near-surface phenomena in lipid bilayer systems compose a new step towards the resolution of the conundrums of fidelity of biomimetic replicas combined with highly instrumentappropriate systems.

The present and future challenges will therefore benefit from an integrated approach, an approach that will be enriching to fundamental knowledge of natural systems as well as for a wide range of synthetic materials and processes in physical surface chemistry.

However the quest for more and more complexity, to better mimic the natural counterparts, should not compromise the controllability of model systems. This is the central challenge that lipid bilayer scientists will have to face in the next decade.

The authors acknowledge financial support from the Italian Ministry for Research, through the program PRIN 2008 (20087K9A2J), FIRB Italnanonet (RBPR05JH2P), and the CSGI.

\section{Notes and references}

1 A. D. Bangham and R. W. Horne, J. Mol. Biol., 1964, 8, 660; A. Tardieu, V. Luzzatti and F. Reman, J. Mol. Biol., 1973, 75, 711.

2 S. Tristram-Nagle and J. F. Nagle, Chem. Phys. Lipids, 2004, 127, 3; J. Katsaras and T. Gutberlet, Lipid Bilayers: Structure and Interactions, Springer, Berlin, 2001.

3 V. P. Torchilin, Nat. Rev. Drug Discovery, 2005, 4, 145; P. Luciani, D. Berti and P. Baglioni, in Colloids in Drug Delivery, ed. M. Fanun, Taylor and Francis CRC Press, Boca Raton, 2009; T. M. Allen and P. R. Cullis, Science, 2004, 303, 1818.

4 S. D. Patil, D. G. Rhodes and D. J. Burgess, AAPS J., 2005, 7, E61; C. R. Safinya, Curr. Opin. Struct. Biol., 2001, 11, 440; S. Chesnoy and L. Huang, Annu. Rev. Biophys. Biomol. Struct., 2000, 29, 27.

5 A. Gomez-Hens and J. M. Fernandez-Romero, $\operatorname{Tr} A C$, Trends Anal. Chem., 2005, 24, 9.

6 V. Noireaux and A. Libchaber, Proc. Natl. Acad. Sci. U. S. A., 2004, 101, 17669; S. M. Christensen and D. Stamou, Soft Matter, 2007, 3, 828; M. Winterhalter, L. Darbois, J. Hemmerle and J. C. Voegel, Langmuir, 2004, 20, 6127; S. Nomura, K. Tsumoto, T. Hamada, K. Akiyoshi and Y. Nakatani, ChemBioChem, 2003, 4, 1172.

7 Y.-H. M. Chan and S. G. Boxer, Curr. Opin. Chem. Biol., 2007, 11, 581; P. A. Janmey and P. K. J. Kinnunen, Trends Cell Biol., 2006, 16, 538 .

8 G. Fragneto and M. Rheinstädter, C. R. Phys., 2007, 8, 865; G. Pabst, N. Kucerka, M.-P. Nieh, M. C. Rheinstädter and J. Katsaras, Chem. Phys. Lipids, 2010, 163, 460.

9 G. Cevc and D. Marsh, Phospholipid Bilayers: Physical Principles and Models, John Wiley and Sons, New York, 1987.

10 H. Wennerström and E. Sparr, Pure Appl. Chem., 2003, 75, 905; R. P. Rand and V. A. Parsegian, Biochim. Biophys. Acta, 1989, 998, 351.

11 Ch. Tanford, in Physics of Amphiphiles: Micelles, Vesicles and Microemulsions, ed. V. Degiorgio and M. Corti, Elsevier Science Bologna, 1985; J. N. Israelachvili, D. J. Mitchell and B. W. Ninham, Biochim. Biophys. Acta, Biomembr., 1977, 470, 185.

12 M. Dubois and T. Zemb, Curr. Opin. Colloid Interface Sci., 2000, 5, 27; S. A. Safran, Statistical Thermodynamics of Surfaces, Interfaces, and Membranes, Addison-Wesley, Reading, 1994; R. R. New, in Liposomes as Tools in Basic Research and Industry, ed. J. R. Philippot and F. Schuber, CRC Press, Boca Raton, 1995; 
M. J. Hope, M. B. Bally, G. Webb and P. R. Cullis, Biochim Biophys. Acta, Biomembr., 1985, 812, 55; D. D. Lasic, Liposomes from Physics to Applications, Elsevier, Amsterdam, 1993; M. J. Ostro, Liposomes from Biophysics to Therapeutics, Marcel Dekker, New York, 1987.

13 V. P. Torchilin and V. Weissig, Liposomes: A Practical Approach, Oxford University Press, 2003; S. C. Basu and M. Basu, Methods in Molecular Biology: Liposomes Methods and Protocols, Humana Press, Totowa, NJ, 2002.

14 E. W. Kaler, A. H. Falls, H. T. Davis, L. E. Scriven and W. G. Miller, J. Colloid Interface Sci., 1982, 90, 424.

15 C. Grabielle-Madelmont, S. Lesieur and M. Ollivon, J. Biochem. Biophys. Methods, 2003, 56, 189; M. Banchelli, F. Baldelli Bombelli, D. Berti and P. Baglioni, Methods Enzymol., 2009, 464, 249.

16 V. M. Trusova and G. P. Gorbenko, Biophys. Chem., 2008, 133, 90; F. Bordi, C. Cametti, S. Sennato and M. Diociaiuti, Biophys. J., 2006, 91, 1513.

17 T. H. Watts, A. A. Brian, J. W. Kappler, P. Marrack and H. McConnell, Proc. Natl. Acad. Sci. U. S. A., 1984, 81, 7564.

18 R. P. Richter, R. Berat and A. R. Brisson, Langmuir, 2006, 22, 3497.

19 E. T. Castellana and P. S. Cremer, Surf. Sci. Rep., 2006, 61, 429.

20 V. Kiessling, M. K. Domanska, D. Murray, C. Wan and L. K. Tamm, in Supported Lipid Bilayers Development and Applications in Chemical Biology, ed. Tadhg P. Begley, Wiley Encyclopedia Of Chemical Biology, John Wiley \& Sons, 2008.

21 E. Kalb, S. Frey and L. K. Tamm, Biochim. Biophys. Acta, Biomembr., 1992, 1103, 307.

22 A. L. Plant, Langmuir, 1993, 9, 2764.

23 M. L. Wagner and L. K. Tamm, Biophys. J., 2001, 81, 266.

24 M. Tanaka and E. Sackmann, Nature, 2005, 437, 656.

25 J. Ekeroth, P. Konradsson and F. Höök, Langmuir, 2002, 18, 7923.

26 Y. Ishii and S. Boxer, J. Am. Chem. Soc., 2003, 125, 3696.

27 C. Rossi and J. Chopineau, Eur. Biophys. J., 2007, 36, 955 and references therein.

28 R. P. Richter and A. R. Brisson, Biophys. J., 2005, 88, 3422; R. P. Richter, A. Mukhopadhyay and A. R. Brisson, Biophys. J., 2003, 85, 3035.

29 B. J. Berne and R. Pecora, Dynamic Light Scattering, Dover Publications, New York, 2000; F. R. Hallet, T. Craig, J. Marsh and B. Nickel, Can. J. Spectros., 1989, 34, 63; R. Pecora, in Soft Matter Characterization, ed. R. Borsali and R. Pecora, Springer, New York, 2008.

30 D. E. Koppel, J. Chem. Phys., 1972, 57, 4814.

31 G. Maulucci, M. De Spirito, G. Arcovito, F. Boffi, A. Congiu Castellano and G. Briganti, Biophys. J., 2005, 88, 3545.

32 M. Banchelli, F. Betti, D. Berti, G. Caminati, F. Baldelli Bombelli, T. Brown, L. M. Wilhelmsson, B. Nordén and P. Baglioni, J. Phys. Chem. B, 2008, 112(35), 10942.

33 P. Lindner and Th. Zemb, Neutrons, X-rays and Light: Scattering Methods Applied to Soft Condensed Matter, Elsevier, Amsterdam, 2002.

34 L. K. Tamm and S. A. Tatulian, Q. Rev. Biophys., 1997, 30, 365 .

35 C. E. Miller, J. Majewski, T. Gog and T. L. Kuhl, Phys. Rev. Lett., 2005, 94, 238104; E. Novakova, K. Giewekemeyer and T. Salditt, Phys. Rev.E: Stat., Nonlinear, Soft Matter Phys., 2006, 74, 051911; M. R. Horton, C. Reich, A. P. Gast, J. O. Radler and B. Nickel, Langmuir, 2007, 23, 6263.

36 R. P. Richter and A. Brisson, Langmuir, 2003, 19, 1632.

37 C. M. McQuaw, L. L. Zheng, A. G. Ewing and N. Winograd, Langmuir, 2007, 23, 5645; M. L. Kraft, P. K. Weber, M. L. Longo, I. D. Hutcheon and S. G. Boxer, Science, 2006, 313, 1948; N. Bourdos, F. Kollmer, A. Benninghoven, M. Sieber and H.-J. Galla, Langmuir, 2000, 16, 1481; N. Bourdos, F. Kollmer, A. Benninghoven, M. Ross, M. Sieber and H.-J. Galla, Biophys. J., 2000, 79, 357.

38 A. Dahlin, M. Zach, T. Rindzevicius, M. Kall, D. S. Sutherland and F. Hook, J. Am. Chem. Soc., 2005, 127, 5043; S. Chah and R. N. Zare, Phys. Chem. Chem. Phys., 2008, 10, 3203-3208.

39 M. L. Kraft, P. K. Weber, M. L. Longo, I. D. Hutcheon and S. G. Boxer, Science, 2006, 313, 1948; E. Reimhult, F. Hook and B. Kasemo, Langmuir, 2003, 19, 1681.
40 A. Janshoff, H.-J. Galla and C. Steinem, Membr. Sci. Technol., 2003, 7, 991; F. Gambinossi, M. Banchelli, A. Durand, D. Berti, T. Brown, G. Caminati and P. Baglioni, J. Phys. Chem. B, 2010, 114(21), 7338 .

41 G. Sauerbrey, Z. Phys., 1959, 155, 206; A. Janshoff, H.-J. Galla and C. Steinem, Angew. Chem., Int. Ed., 2000, 39, 4004; M. V. Voinova, M. Rodahl, M. Jonson and B. Kasemo, Phys. Scr., 1999, 59, 391.

42 F. Hook, B. Kasemo, T. Nylander, C. Fant, K. Sott and H. Elwing, Anal. Chem., 2001, 73, 5796.

43 J. M. Johnson, T. Ha, S. Chu and S. G. Boxer, Biophys. J., 2002, 83, 3371.

44 B. Cannon, N. Weaver, Q. S. Pu, V. Thiagarajan, S. R. Liu, J. Y. Huang, M. W. Vaughn and K. H. Cheng, Langmuir, 2005, 21, 9666.

45 N. Kucerka, J. Pencer, J. N. Sachs, J. F. Nagle and J. Katsaras, Langmuir, 2007, 23, 1292.

46 G. Palazzo, A. Mallardi, M. Giustini, D. Berti and G. Venturoli, Biophys. J., 2000, 79, 1171; L. Ambrosone, A. Mallardi, G. Palazzo and G. Venturoli, Phys. Chem. Chem. Phys., 2002, 4, 3071 .

47 F. Albertorio, A. J. Diaz, T. Yang, V. A. Chapa, S. Kataoka, E. T. Castellana and P. S. Cremer, Langmuir, 2005, 21, 7476.

48 D. Berti, Curr. Opin. Colloid Interface Sci., 2006, 11, 74.

49 D. Berti, F. Baldelli Bombelli, M. Fortini and P. Baglioni, J. Phys. Chem. B, 2007, 111, 11734; S. Milani, F. Baldelli Bombelli, D. Berti and P. Baglioni, J. Am. Chem. Soc., 2007, 129, 11664; F. Baldelli Bombelli, D. Berti, F. Pini, U. Keiderling and P. Baglioni, J. Phys. Chem. B, 2004, 108, 16427.

50 M. Banchelli, F. Gambinossi, A. Durand, G. Caminati, T. Brown, D. Berti and P. Baglioni, J. Phys. Chem. B, 2010, 114, 7348.

51 A. Gissot, C. Di Primo, I. Bestel, G. Giannone, H. Chapuis and P. Barthelemy, Chem. Commun., 2008, 5550; F. Betti, F. Baldelli Bombelli, F. Gambinossi, G. Caminati, T. Brown, P. Baglioni and D. Berti, Soft Matter, 2009, 5, 163; F. Baldelli Bombelli, F. Gambinossi, M. Lagi, D. Berti, G. Caminati, T. Brown, F. Sciortino, B. Nordén and P. Baglioni, J. Phys. Chem. B, 2008, $112(48), 15283$.

52 K. Simons and E. Ikonen, Nature, 1997, 387, 569; K. Simons and W. L. C. Vaz, Annu. Rev. Biophys. Biomol. Struct., 2004, 33, 269; K. Simons and M. J. Gerl, Nat. Rev. Mol. Cell Biol., 2010, 11, 688; H.-J. Galla, E. Sackmann, H. J. Galla and E. Sackmann, J. Am. Chem. Soc., 1975, 97, 4114.

53 E. London, Biochim. Biophys. Acta, Mol. Cell Res., 2005, 1746, 203.

54 J. Zimmerberg and M. M. Kozlov, Nat. Rev. Mol. Cell Biol., 2005, 7, 9; W. B. Huttner and J. Zimmerberg, Curr. Opin. Cell Biol., 2001, 13, 478; H. T. McMahon and J. L. Gallop, Nature, 2005, 438, 590; A. Roux, D. Cuvelier, P. Nassoy, J. Prost, P. Bassereau and B. Goud, EMBO J., 2005, 24, 1537.

55 R. Ehehalt, P. Keller, C. Haass, C. Thiele and K. Simons, J. Cell Biol., 2003, 160, 113; K. Matsuzaki, Biochim. Biophys. Acta, 2007, 1768, 1942; K. Simons and R. Ehehalt, J. Clin. Invest., 2002, $110,597$.

56 J. Korlach, P. Schwille, W. W. Webb and G. W. Feigenson, Proc. Natl. Acad. Sci. U. S. A., 1999, 96, 8461; L. A. Bagatolli and E. Gratton, Biophys. J., 2000, 78, 290.

57 G. W. Feigenson and J. T. Buboltz, Biophys. J., 2001, 80, 2775.

58 J. Pencer, T. Mills, V. N. P. Anghel, S. Krueger, R. M. Epand and J. Katsaras, Eur. Phys. J., 2005, 18, 447; J. Pencer, V. N. P. Anghel, N. Kucerka and J. Katsaras, J. Appl. Crystallogr., 2006, 39, 791.

59 J. Pencer, V. N. P. Anghel, N. Kucerka and J. Katsaras, J. Appl. Crystallogr., 2007, 40, 771

60 J. Yuan, A. Kiss, Y. H. Pramudya, L. T. Nguyen and L. S. Hirst, Phys. Rev. E: Stat., Nonlinear, Soft Matter Phys., 2009, 79, 031924.

61 W. Lin, C. Craig, D. Blanchette, T. V. Ratto and M. L. Longo, Biophys. J., 2006, 90, 228.

62 M. Sundha, S. Svedhemb and D. S. Sutherland, Phys. Chem. Chem. Phys., 2010, 12, 453.

63 K. Athmakuri, C. Padala, J. Litt, R. Cole, S. Kumar and R. S. Kane, Langmuir, 2010, 26, 397.

64 C. Reich, M. R. Horton, B. Krause, A. P. Gast, J. O. Radler and Bert Nicke, Biophys. J., 2008, 95, 657.

65 V. Kiessling, C. Wan and L. K. Tamm, Biochim. Biophys. Acta, Biomembr., 2009, 1788, 64. 
66 R. P. Richter, N. Maury and A. R. Brisson, Langmuir, 2005, 21, 299.

67 S. Morandi, M. Puggelli and G. Caminati, Colloids Surf., A, 2008, 321, 125.

68 N. Kucerka, M.-P. Nieh and J. Katsaras, Langmuir, 2009, 25, 13522 .
69 M. J. Hope, T. E. Redelmeier, K. F. Wong, W. Rodriguez and P. R. Cullis, Biochemistry, 1989, 28, 4181.

70 S. Pautot, B. J. Frisken and D. A. Weitz, Proc. Natl. Acad. Sci. U. S. A., 2003, 100, 10718.

71 H. T. Cheng, Megha and E. London, J. Biol. Chem., 2008, 284, 6079 . 